\title{
Automatic contactless injection, transportation, merging, and ejection of droplets with a multifocal point acoustic levitator $\odot$
}

Cite as: Rev. Sci. Instrum. 89, 125105 (2018); https://doi.org/10.1063/1.5063715

Submitted: 30 September 2018 . Accepted: 12 November 2018 . Published Online: 10 December 2018

Marco A. B. Andrade (D), Thales S. A. Camargo, and Asier Marzo (D)

\section{COLLECTIONS}

This paper was selected as Featured
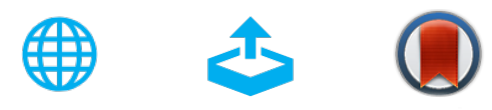

\section{ARTICLES YOU MAY BE INTERESTED IN}

TinyLev: A multi-emitter single-axis acoustic levitator

Review of Scientific Instruments 88, 085105 (2017); https://doi.org/10.1063/1.4989995

Nonlinear trapping stiffness of mid-air single-axis acoustic levitators

Applied Physics Letters 113, 034102 (2018); https://doi.org/10.1063/1.5034116

Realization of compact tractor beams using acoustic delay-lines

Applied Physics Letters 110, 014102 (2017); https://doi.org/10.1063/1.4972407

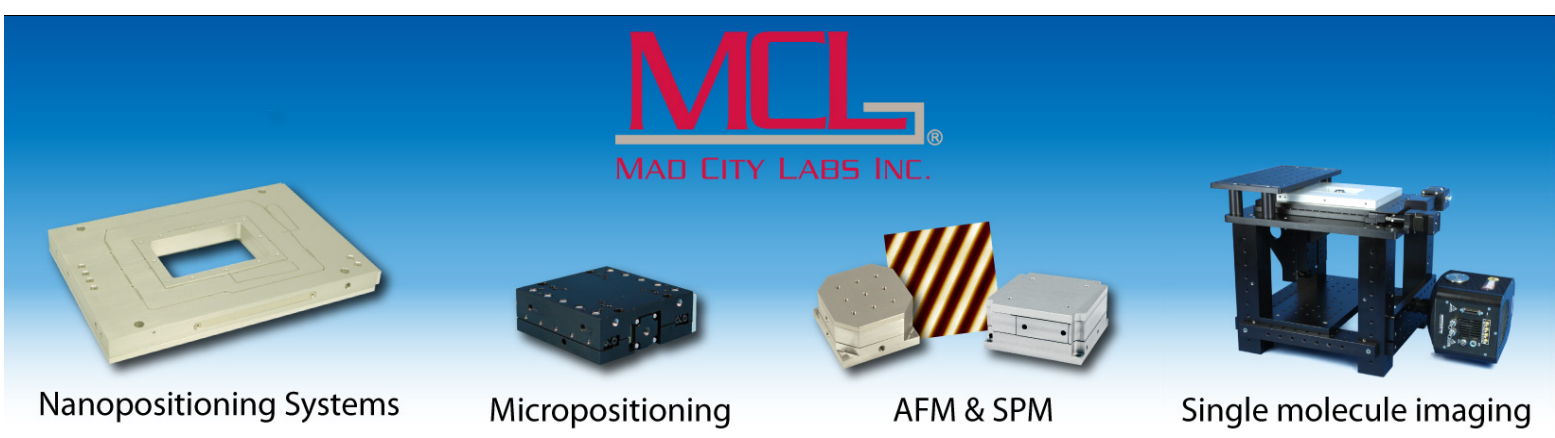




\title{
Automatic contactless injection, transportation, merging, and ejection of droplets with a multifocal point acoustic levitator
}

\author{
Marco A. B. Andrade, ${ }^{1, a)}$ Thales S. A. Camargo, ${ }^{2}$ and Asier Marzo ${ }^{3}$ \\ ${ }^{1}$ Institute of Physics, University of São Paulo, São Paulo 05508-090, Brazil \\ ${ }^{2}$ Department of Mining and Petroleum Engineering, University of São Paulo, Santos 11013-560, Brazil \\ ${ }^{3}$ Computer Science, Public University of Navarre, Pamplona 31006, Navarre, Spain
}

(Received 30 September 2018; accepted 12 November 2018; published online 10 December 2018)

\begin{abstract}
We present an acoustic levitation system that automatically injects, transports, merges and ejects liquid droplets in mid-air. The system consists of a phased array operating at $40 \mathrm{kHz}$ on top of a plane reflector. The phase array generates multiple focal points at independent positions that form standing waves between the array and the reflector. In the reflector there is an inlet for a piezoelectric droplet injector which automatically inserts liquid droplets at the lower pressure nodes of the standing waves, and a hole that serves as an outlet for ejecting the processed droplets out of the system. Simulations of the acoustic radiation potential acting on the levitating droplets are in good agreement with the experiments. High-speed footage captured the functioning of the system in four fluidic operations: injection, transport, merging and ejection of liquid droplets. Having these operations integrated reliably into a single automatic system paves the way for the adoption of mid-air acoustophoretic processing in biological, chemical and pharmaceutical applications. Published by AIP Publishing. https://doi.org/10.1063/1.5063715
\end{abstract}

\section{INTRODUCTION}

Manipulating small volumes of liquids has a vital role in various disciplines such as biology, chemistry and pharmacy. This importance is conveyed by the typical depiction of a laboratory, which in most occasions showcases a person holding a pipette to transport and mix liquids. With the advancement of liquid-handling technology, new methods for automatically manipulating fluids have been developed. One of the state-of-the-art techniques is based on electrowetting, in which an array of electrodes is employed for manipulating liquid droplets over a hydrophobic surface. ${ }^{1-3}$ With this technique, different operations can be performed, such as dispensing, transporting, merging, splitting and mixing. ${ }^{1,2}$ However, a fundamental drawback is that the liquids are in contact with the surface, leading to cross-contamination. This problem can be avoided by using contactless handling methods, such as acoustic levitation. ${ }^{4,5}$

Acoustic levitation employs acoustic radiation forces 6 to suspend objects in mid-air. Unlike other levitation techniques, such as optical tweezers ${ }^{7}$ or electrostatic manipulation, ${ }^{8}$ acoustic levitation supports a wide range of materials, including solids, ${ }^{9-11}$ liquids, ${ }^{12-14}$ bubbles, ${ }^{15,16}$ and small living animals. ${ }^{17,18}$ This feature makes acoustic levitation a desirable tool for analytical chemistry, ${ }^{19,20}$ biology ${ }^{21}$ or pharmacy. $^{22}$

In the recent years, different acoustic approaches have been proposed to manipulate liquid droplets. ${ }^{14,23-28}$ Koyama and Nakamura ${ }^{23}$ developed a levitation system formed by a plane reflector and a metal plate attached to transducers at its ends. By changing the relative phase between the

\footnotetext{
a) Author to whom correspondence should be addressed: marcobrizzotti@ gmail.com
}

transducers, they could transport ethanol droplets. Foresti et $a l .{ }^{14}$ presented a levitation platform to manipulate and merge liquid droplets in mid-air. They generated a standing wave between multiple Langevin transducers and a reflector on top, and the levitating droplets could be moved and merged by modulating the amplitude of subsequent transducers. Ochiai et al. ${ }^{24}$ employed four arrays arranged in a cube to manipulate small solid objects and liquid droplets in three-dimensions. Recently, Watanabe et al. ${ }^{28}$ used a $7 \times 7$ square array of $40 \mathrm{kHz}$ transducers and an opposing reflector to levitate and merge two liquid droplets. In their system, the horizontal positions of the droplets were controlled by rapidly multiplexing (i.e. $500 \mathrm{~Hz}$ ) a focal point between the two positions.

Despite the recent progress in manipulating liquid droplets by acoustic levitation methods, a remaining issue is how to insert and remove the samples from the levitator. In the state-of-the-art levitation systems, ${ }^{11,14,22,23,28}$ samples are manually inserted and removed with a syringe, requiring a skilled person to avoid sample losses and preventing the automation of the processes. Therefore, executing different fluidic operations such as injection, ${ }^{19,29,30}$ transportation, ${ }^{14,23,24}$ merging $^{14,26,28}$ and ejection ${ }^{31}$ in a single levitation device would open up multiple possibilities, enabling the automatic processing of droplets in mid-air.

In this article, we present an integrated acoustic levitation system that automatically injects, transports, merges and ejects liquid droplets in mid-air. These operations are captured using a high-speed camera and numerical simulations are carried out to predict the potential of the acoustic radiation force acting on the levitated droplets. The manipulation of the droplets is achieved using a phased-array made of commercially available and inexpensive ultrasonic transducers that generate multiple focal points simultaneously. 

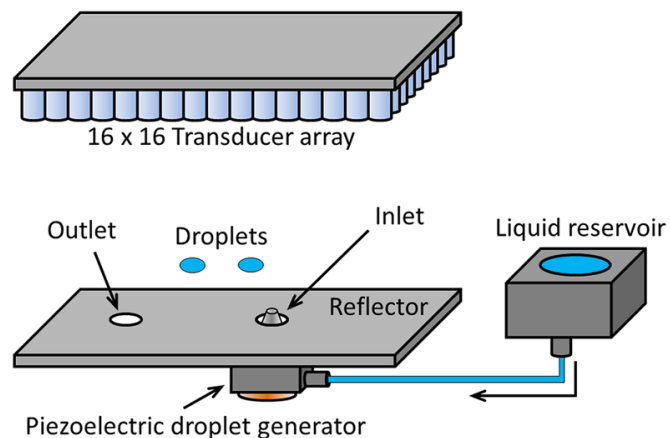

FIG. 1. Acoustic levitation system made of a $16 \times 16$ transducers array and a plane reflector. Liquid droplets are inserted by a piezoelectric droplet generator. The droplet outlet consists of a circular hole of $3.5 \mathrm{~mm}$ in diameter.

\section{ACOUSTIC LEVITATION SYSTEM}

The acoustic levitation system (Fig. 1) consists of a $16 \times 16$ array of $40 \mathrm{kHz}$ ultrasonic transducers of $9.8 \mathrm{~mm}$ diameter (Manorshi MSO-P1040H07T) and a plane reflector, which are separated by a distance of $110 \mathrm{~mm}$. The separation distance between the phased array and the reflector was selected by focusing the array without the reflector at different positions (center, side and corner) and determining the pressure amplitude as a function of the distance to the array (Fig. 2). In general, a distance of $110 \mathrm{~mm}$ [black dashed line of Fig. 2(b)] provides the maximum amplitude for the central, side and corner focal point positions. This distance is still optimum when the reflector is included since it just acts as a mirror.

The emission phase of each transducer is controlled by a computer, which communicates with the driver board via serial communication (UART protocol $250 \mathrm{kBauds}$ ). The driver board uses a Field-programmable gate array (FPGA) (Altera Cyclone IV-EP4CE6) to generate the control signals. 1-to-8 serial to parallel shift registers (TI 74hc595) multiplex 32 IOs of the FPGA into 256 independent digital channels. Mosfet Drivers (Microchip MIC4127) amplify the signals up to $20 \mathrm{Vpp}$ half-square waves, that are fed into the transducers. Due to the narrowband nature of the transducers, the output pressure is sinusoidal. ${ }^{32}$ The array provided a phase emission resolution of $\pi / 16 \mathrm{rad}$. To independently control the levitation position of two liquid droplets, the transducers are divided into two sets, forming a chess pattern [Fig. 4(a) (Multimedia view)], with each set of transducers generating a different focus. By setting the focus point at the reflector surface [red and blue circles in Figs. 4(b) and 4(c) (Multimedia view)], the superposition of the incident and reflected waves forms a standing wave. The standing wave has a series of pressure nodes where liquid droplets can be trapped and moved in two dimensions above the surface if the standing wave is translated. The lowest nodes are located $2.5 \mathrm{~mm}$ above the reflector and are employed for levitation.

The acoustic levitation system is suitable for levitating and manipulating liquid droplets ranging from hundreds of micrometers to few millimeters in diameter. The maximum droplet diameter is limited by the acoustic wavelength of the standing wave (in general, droplets of diameters up to onehalf of the wavelength can be levitated), whereas the minimum droplet size is restricted by the drag forces caused by acoustic streaming. ${ }^{33}$

The system has an integrated droplet injector inserted in the reflector. The injector consists of a low-cost droplet generator $^{34}$ formed by two main parts: a liquid reservoir, and a fluid chamber with a piezoelectric buzzer and a nozzle with an outlet diameter of $1 \mathrm{~mm}$. The nozzle tip is inserted pointing upwards in a small hole $(5.5 \mathrm{~mm}$ in diameter) in the reflector. When a square pulse ( $60 \mathrm{Vpp}, 1 \mathrm{~ms}$ pulse width) is applied to the piezoelectric element of the droplet generator, a droplet is injected and trapped at the bottom pressure node of the standing wave.

The droplet outlet consists of a circular orifice $(3.5 \mathrm{~mm}$ diameter) in the reflector. The procedure to eject the levitating droplet consists in moving the droplet above the outlet orifice and then switching the acoustic field off. When there is no standing wave to trap the droplet, it falls through the orifice due to gravity. The diameter of the outlet was selected through experimental tests. The circular orifice is large enough to avoid collisions with the falling droplet and sufficiently small to reflect most of the incoming wave.

\section{NUMERICAL SIMULATION}

The pressure and potential field generated by the levitation system is simulated by combining a modified matrix method $^{35,36}$ with the Gor'kov potential equation. ${ }^{37}$ In the traditional matrix method, ${ }^{35,36}$ the radiation surface of each transducer is discretized in small surface elements. Herein, we
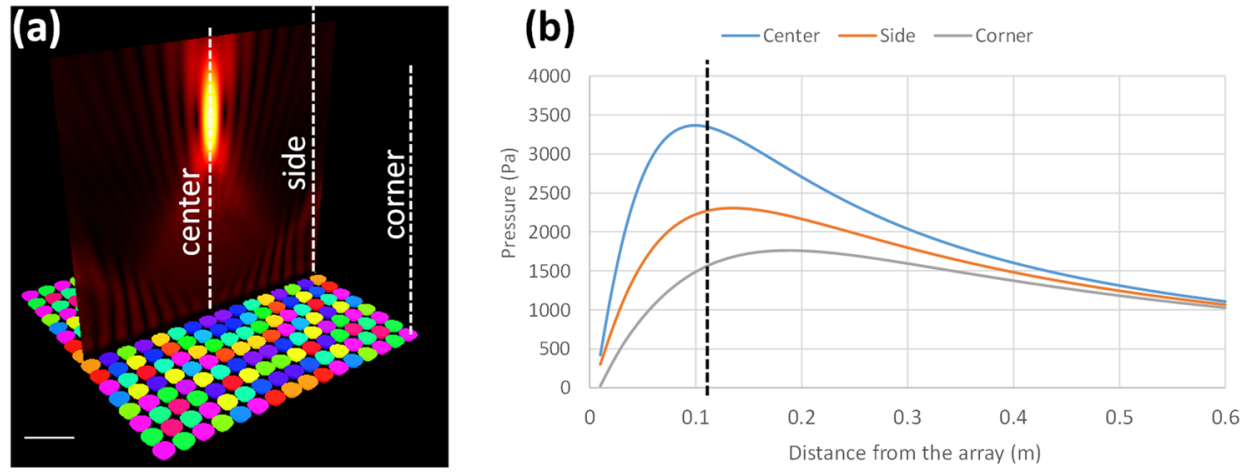

FIG. 2. (a) Simulated amplitude pressure distribution of the phased-array generating a focal point $110 \mathrm{~mm}$ above its center. (b) Simulated amplitude pressure generated at focal points produced at different distances at the center, side and corner of the array. The distance of $0.11 \mathrm{~m}$ was selected as it provides the overall largest pressure.

Amplitude: 0 - $3500 \mathrm{~Pa}$

Emission Phase: $-\pi$ to $\pi$ 


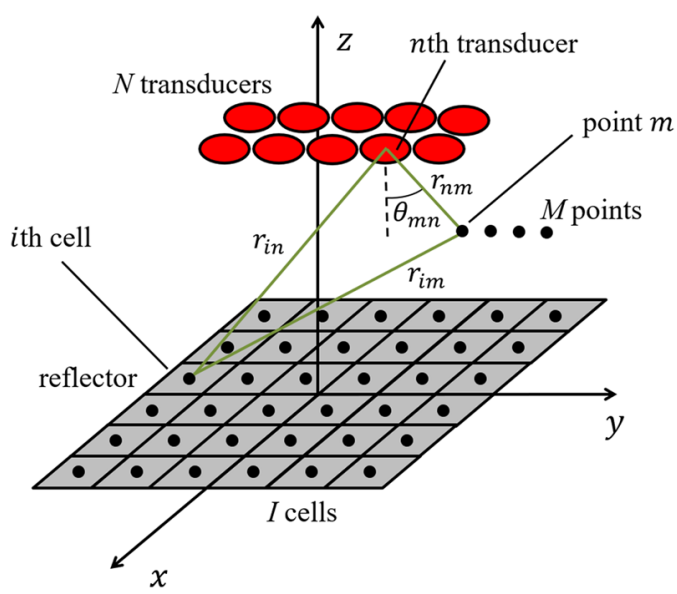

FIG. 3. Schematic of the modified matrix method in which $N$ transducers emit as circular piston sources, a reflector is below them and the acoustic pressure is calculated at $M$ points.

use an analytical expression for the acoustic pressure generated by a circular piston in the far field.

Figure 3 illustrates the parameters of the modified matrix method. We are interested in determining the complex pressure $p_{m}$ at a set of $M$ points located between $N$ circular transducers and a plane reflector.

Without taking into account the reflections from the reflector, the direct acoustic pressure $p_{m}$ at a point $m$, generated by the $n$th transducer is given by ${ }^{38}$

$$
p_{m}=-\frac{a^{2} \rho_{0} \omega^{2} \delta_{n}}{2 r_{n m}}\left[\frac{2 J_{1}\left(k a \sin \theta_{n m}\right)}{k a \sin \theta_{n m}}\right] e^{-j k r_{n m}},
$$

where $j=\sqrt{-1}, a$ is the transducer radius, $r_{n m}$ is the distance from the $n$th transducer to the point $m$, the angle $\theta_{n m}$ is defined in Fig. 3, $J_{1}$ denotes the first-order Bessel function of first kind, $k$ is the wavenumber, and $c_{0}=343 \mathrm{~m} / \mathrm{s}$ and $\rho_{0}=1.2 \mathrm{~kg} / \mathrm{m}^{3}$ corresponds to the sound velocity and density of air, respectively. In Eq. (1), $\delta_{n}$ is the complex displacement of the transducer face, where the magnitude of $\delta_{n}$ represents the displacement amplitude and the argument of the complex number corresponds to the phase.

For the case of $N$ transducers, the direct acoustic pressure at the set of points $m$ can be calculated by the superposition of the waves emitted by each transducer. In a matrix form, the acoustic pressure at a set of points can be expressed by

$$
\left[\begin{array}{c}
p_{1} \\
p_{2} \\
\vdots \\
p_{M}
\end{array}\right]=\left(\frac{\omega \rho_{0} c_{0}}{\lambda}\right)\left[\begin{array}{cccc}
T_{11}^{T M} & T_{12}^{T M} & \ldots & T_{12}^{T M} \\
T_{21}^{T M} & T_{22}^{T M} & \ldots & T_{2 N}^{T M} \\
\vdots & \vdots & \ddots & \vdots \\
T_{M 1}^{T M} & T_{M 2}^{T M} & \ldots & T_{M N}^{T M}
\end{array}\right]\left[\begin{array}{c}
\delta_{1} \\
\delta_{2} \\
\vdots \\
\delta_{N}
\end{array}\right],
$$

where the elements of the transfer matrix from the transducers to the points $\left(\mathbf{T}^{\mathbf{T M}}\right)$ are calculated by

$$
T_{m n}^{T M}=-\frac{a^{2} \pi}{r_{n m}}\left[\frac{2 J_{1}\left(k a \sin \theta_{n m}\right)}{k a \sin \theta_{n m}}\right] e^{-j k r_{n m}},
$$

Equations (2) and (3) are essentially the same as used by Marzo et al. ${ }^{11,27}$ to calculate the acoustic pressure field generated by an array of transducers. To introduce the first wave reflected at the reflector, we add a reflection term to Eq. (2), such that the acoustic pressure at the $M$ points can be written in the following matrix form

$$
\mathbf{P}=\left(\frac{\omega \rho_{0} c_{0}}{\lambda}\right) \mathbf{T}^{\mathbf{T M}} \boldsymbol{\delta}+\left(\frac{j \omega \rho_{0} c_{0}}{\lambda^{2}}\right) \mathbf{T}^{\mathbf{R} \mathbf{M}} \mathbf{T}^{\mathbf{T R}} \boldsymbol{\delta},
$$

where $\mathbf{P}=\left[\begin{array}{lll}p_{1} & p_{2} \ldots p_{M}\end{array}\right]^{T}$ is the pressure vector containing the complex pressure at the set of points $m, \boldsymbol{\delta}=\left[\begin{array}{llll}\delta_{1} & \delta_{2} & \ldots & \delta_{N}\end{array}\right]^{T}$ is the displacement vector containing the complex displacement amplitude (i.e. amplitude and phase) of each transducer, $\mathbf{T}^{\mathbf{R M}}$ is the transfer matrix from the reflector cells to the measurement points, and $\mathbf{T}^{\mathbf{T R}}$ corresponds to the transfer matrix from the transducers to the reflector cells. The elements of these matrices are calculated by ${ }^{35,36}$

$$
\begin{gathered}
T_{m i}^{R M}=\frac{s_{i}}{r_{i m}} e^{-j k r_{i m}}, \\
T_{i n}^{T R}=-\frac{a^{2} \pi}{r_{i n}}\left[\frac{2 J_{1}\left(k a \sin \theta_{i n}\right)}{k a \sin \theta_{i n}}\right] e^{-j k r_{i n}} .
\end{gathered}
$$

In Eq. (5), $s_{i}$ is the area of the $i$ th cell of the reflector. Equation (4) is used to obtain the acoustic pressure in a set of points considering the direct wave and the first reflection from the reflector. Normally, the pressure distribution is determined in a rectangular grid consisting of $M$ points.

From the acoustic pressure $p$, the particle velocity distribution $\boldsymbol{u}$ is calculated with the following equation: ${ }^{38}$

$$
\boldsymbol{u}=-\frac{\nabla p}{j \omega \rho_{0}} .
$$

This matrix method is used to calculate the acoustic pressure distribution $p$ and the particle velocity distribution $\boldsymbol{u}$ between the array and the reflector. The simulation considers the superposition of the emitted wave by the array and the first reflection from the reflector. The pressure and particle velocity fields obtained by the matrix method is then replaced in the Gor'kov equation to find the potential of the acoustic radiation force acting on the levitating droplets. This potential, also referred as Gor'kov potential, is calculated by ${ }^{37}$

$$
U=2 \pi R^{3}\left(\frac{1}{3 \rho_{0} c_{0}^{2}}\left\langle p^{2}\right\rangle-\frac{\rho_{0}}{2}\langle\boldsymbol{u} \cdot \boldsymbol{u}\rangle\right),
$$

where $R$ is the radius of the liquid droplets and the angle brackets \langle\rangle represent the time average. Neglecting gravity, the position of the levitated object should coincide with the positions of minimum Gor'kov potential.

\section{RESULTS}

The matrix method was used to calculate the acoustic pressure distribution and the Gor'kov potential between the transducers and the reflector. It was considered that the origin of the coordinates system is in the center of the reflector and the transducer array is located $110 \mathrm{~mm}$ above the reflector. The droplet inlet is located at $(x, y, z)=(-3.0 \mathrm{~mm}, 0,0)$ and the outlet is located at $(-25.3 \mathrm{~mm}, 0,0)$.

A simulation is presented in Fig. 4 (Multimedia view), with the first set of transducers (red) focused at $(-19.7 \mathrm{~mm}$, $0,0)$ and the second set (blue) focused at $(-7.2 \mathrm{~mm}, 0,0)$. This 

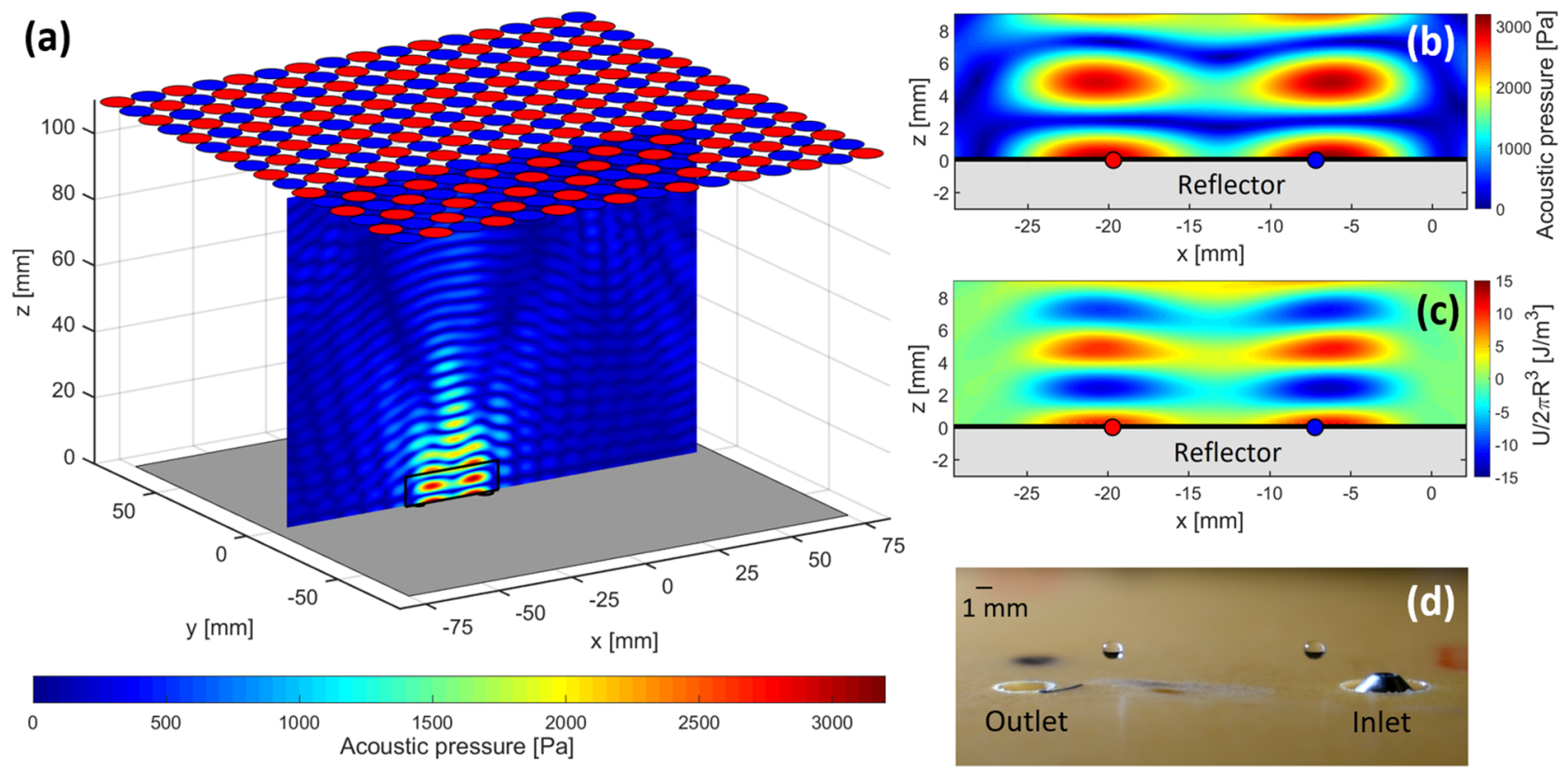

FIG. 4. Comparison between simulation and experimental levitation positions of two water droplets. The transducers of set 1 (red) are focusing at ( $-19.7 \mathrm{~mm}$, 0,0 ) and set 2 (blue) is focused at $(-7.2 \mathrm{~mm}, 0,0)$. (a) Simulated acoustic pressure distribution over the xz-plane, zoomed-in at (b). (c) Simulated Gor'kov potential. (d) Picture of the levitating droplets. A video showing the simulated Gor'kov potential and the droplets manipulation is available online. Multimedia view: https://doi.org/10.1063/1.5063715.1

leads to Gor'kov potential minima located above the focus points, in which liquid droplets can be levitated. Figure 4(d) (Multimedia view) shows two levitating water droplets for this configuration. The experimental levitating positions are in good agreement with the positions of minimum Gor'kov potential.

The automatic manipulation capabilities of our system are demonstrated by programming and executing the following sequence of fluidic operations: (1) injection of the first droplet, (2) transport of this droplet from $x=-4.5 \mathrm{~mm}$ to $x=-20 \mathrm{~mm}$, (3) injection of a second droplet, (4) transport and merging of the two droplets at $x=-16 \mathrm{~mm}$, (5) transport and ejection of the merged droplet to the outlet (see Multimedia view in Fig. 4). This sequence of operations was recorded by a high-speed camera (Photron FASTCAM MINI UX50) at 250 FPS, and a tracking algorithm was used to detect the center of each droplet over time. The $x$-position of the droplets throughout the injection, transportation, mixing and ejections are shown in Fig. 5. It can be observed that there is good agreement between the simulated potential minima and the experimental positions of the levitated droplets. It can also be seen that before the droplets collision at $t=25.3 \mathrm{~s}$, there is an attractive acceleration between the droplets. As observed by Foresti et al. ${ }^{14}$ this acceleration is caused by the secondary acoustic radiation force ${ }^{39}$ in which the wave scattered by one droplet causes an attractive force on the other droplet.

The injection, merging, and ejection operations were also captured at a higher frame rate (i.e. 10000 FPS) and are shown in Fig. 6 as well as in its Multiemedia view. After the droplet is injected, it oscillates around the potential minimum with a peak-to-peak amplitude of $3 \mathrm{~mm}$. This oscillation persists for few seconds, until it is damped by the drag forces of air and the droplet reaches the equilibrium position (i.e., the position of minimum potential). As described in the literature ${ }^{40-42}$ the acoustic radiation force acting on the droplets can be approximated by the restoring force of a spring and the oscillations can be described by making an analogy with a damped harmonic oscillator.

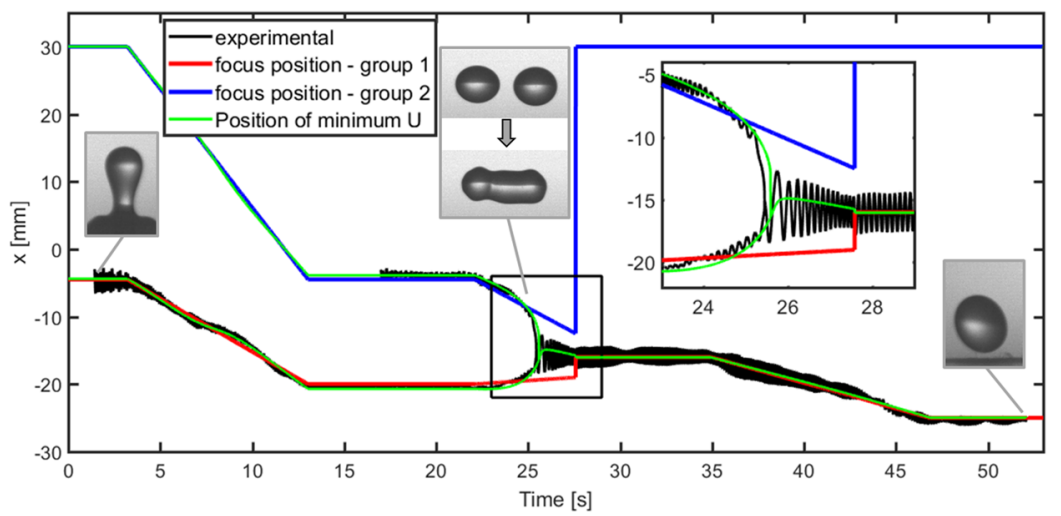

FIG. 5. X-position of the droplets over time. The first droplet is injected in the levitator at $t=1.4 \mathrm{~s}$ and the second 1 at $t=17 \mathrm{~s}$. The focus positions of both sets of transducers are controlled so that the two droplets are transported and merged at $t=25.4 \mathrm{~s}$. Then, the merged droplet is transported to the outlet located at $x=-25.3 \mathrm{~mm}$. At $t=52 \mathrm{~s}$, the levitator is switched off, making the droplet fall through the outlet. The inset shows in more detail the droplets $\mathrm{x}$-position before and after the merging operation. 


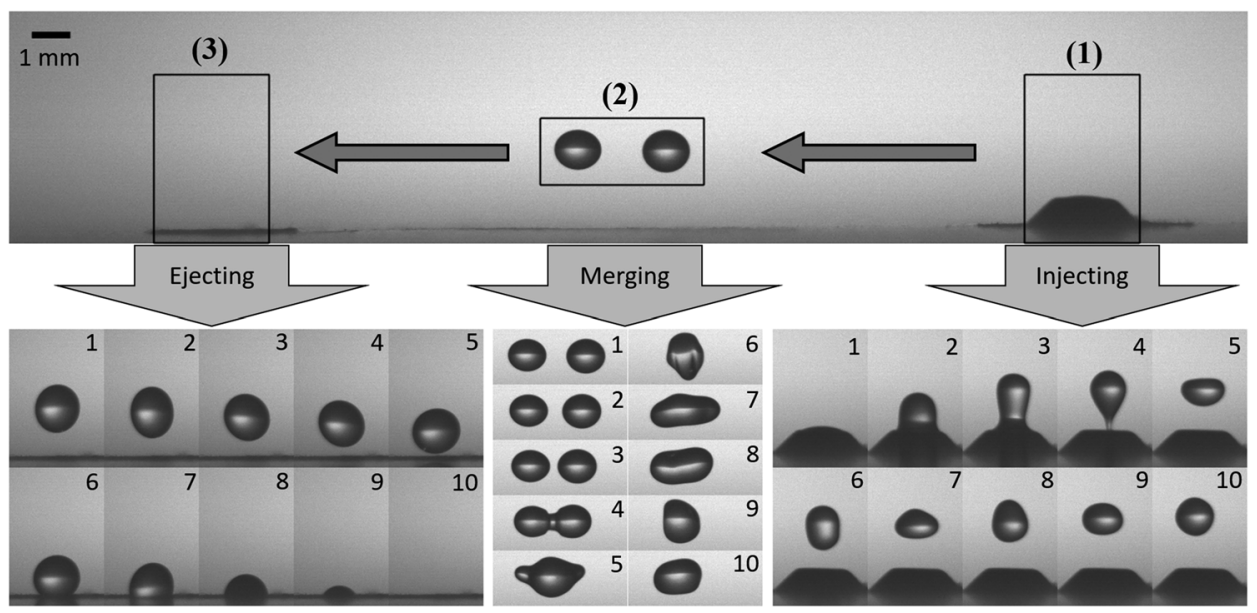

FIG. 6. Injection (1), merging (2) and ejection (3) operations. The time between frames is $2 \mathrm{~ms}$. A video showing these operations is available online. Multimedia view: https://doi.org/10.1063/1.5063715.2
The merging operation was achieved by a procedure similar to that described by Watanabe et al. ${ }^{28}$ but instead of generating the focal points by rapidly switching the phases of all the transducers, our focusing scheme divided the transducers into two sets to obtain two simultaneous and independent focus points; i.e. Watanabe et al. employed time multiplexing whereas we have used spatial multiplexing. Finally, the droplet ejection operation is presented on the left-hand side of Fig. 6 (Multimedia view), which shows the droplet falling through the circular orifice due to the action of gravity.

\section{CONCLUSION}

We have presented an integrated acoustic levitation system that can be used for the automatic contactless injection, transportation, merging and ejection of liquid droplets in mid-air. To manipulate the droplets, it uses multiple focal points generated with a phased array made of inexpensive and commercially available ultrasonic transducers. A numerical method combining a matrix method with the Gor'kov equation was used for calculating the potential of the acoustic radiation force acting on the droplets, showing that the positions of minimum potential agree with the experimental droplet positions. Although the current system has only one inlet and one outlet, it can easily be extended by adding more inlets and outlets. It is also possible to modify our focusing scheme to generate more focal points, allowing the simultaneous manipulation of a larger number of droplets. We expect that the presented system will enable designs that provide reliable and automatic contactless manipulation in the areas of biology, analytical chemistry and pharmacy.

\section{ACKNOWLEDGMENTS}

This research was supported by the São Paulo Research Foundation-FAPESP (Grants Nos. 2017/27078-0 and 2018/04101-0).

${ }^{1}$ K. Choi, A. H. C. Ng, R. Fobel, and A. R. Wheeler, Annu. Rev. Anal. Chem. 5, $413(2012)$

${ }^{2}$ S. K. Cho, H. Moon, and C. J. Kim, J. Microelectromech. Syst. 12, 70 (2003).

${ }^{3}$ M. Abdelgawad and A. R. Wheeler, Adv. Mater. 21, 920 (2009).
${ }^{4}$ E. H. Brandt, Nature 413, 474 (2001)

${ }^{5}$ M. A. B. Andrade, N. Pérez, and J. C. Adamowski, Braz. J. Phys. 48, 190 (2018).

${ }^{6}$ H. Bruus, Lab Chip 12, 1014 (2012).

${ }^{7}$ D. G. Grier, Nature 424, 810 (2003).

${ }^{8}$ W. K. Rhim, S. K. Chung, D. Barber, K. F. Man, G. Gutt, A. Rulison, and R. E. Spjut, Rev. Sci. Instrum. 64, 2961 (1993).

${ }^{9}$ W. J. Xie and B. Wei, Phys. Rev. E 66, 026605 (2002).

${ }^{10}$ M. A. B. Andrade, F. C. Buiochi, and J. Adamowski, IEEE Trans. Ultrason. Ferroelectrics Freq. Control 57, 469 (2010).

${ }^{11}$ A. Marzo, S. A. Seah, B. W. Drinkwater, D. R. Sahoo, B. Long, and S. Subramanian, Nat. Commun. 6, 8661 (2015).

${ }^{12}$ A. L. Yarin, M. Pfaffenlehner, and C. Tropea, J. Fluid Mech. 356, 65 (1998).

${ }^{13}$ D. Zang, Y. Yu, Z. Chen, X. Li, H. Wu, and X. Geng, Adv. Colloid Interface Sci. 243, 77 (2017).

${ }^{14}$ D. Foresti, M. Nabavi, M. Klingauf, A. Ferrari, and D. Poulikakos, Proc. Natl. Acad. Sci. U. S. A. 110, 12549 (2013).

${ }^{15}$ D. Zang, K. Lin, L. Li, Z. Chen, X. Li, and X. Geng, Appl. Phys. Lett. 110, 121602 (2017).

${ }^{16}$ D. Zang, L. Li, W. Di, Z. Zhang, C. Ding, Z. Chen, W. Shen, B. P. Binks, and X. Geng, Nat. Commun. 9, 3546 (2018).

${ }^{17}$ W. J. Xie, C. D. Cao, Y. J. Lü, Z. Y. Hong, and B. Wei, Appl. Phys. Lett. 89, 214102 (2006).

${ }^{18}$ M. Sundvik, H. J. Nieminen, A. Salmi, P. Panula, and E. Hæggström, Sci. Rep. 5, 13596 (2015).

${ }^{19}$ S. Santesson and S. Nilsson, Anal. Bioanal. Chem. 378, 1704 (2004).

${ }^{20}$ F. Priego-Capote and L. de Castro, TrAC, Trends Anal. Chem. 25, 856 (2006).

${ }^{21}$ A. Scheeline and R. L. Behrens, Biophys. Chem. 165-166, 1 (2012).

${ }^{22}$ C. J. Benmore and J. K. R. Weber, Phys. Rev. X 1, 011004 (2011).

${ }^{23}$ D. Koyama and K. Nakamura, IEEE Trans. Ultrason. Ferroelectrics Freq. Control 57, 1152 (2010).

${ }^{24}$ Y. Ochiai, T. Hoshi, and J. Rekimoto, PLoS One 9, e97590 (2014).

${ }^{25}$ R. Nakamura, Y. Mizuno, and K. Nakamura, Jpn. J. Appl. Phys. 52, 07 HE02 (2013).

${ }^{26}$ N. Bjelobrk, M. Nabavi, and D. Poulikakos, J. Appl. Phys. 112, 053510 (2012).

${ }^{27}$ A. Marzo, A. Barnes, and B. W. Drinkwater, Rev. Sci. Instrum. 88, 085105 (2017).

${ }^{28}$ A. Watanabe, K. Hasegawa, and Y. Abe, Sci. Rep. 8, 10221 (2018).

${ }^{29}$ E. T. Chainani, W. H. Choi, K. T. Ngo, and A. Scheeline, Anal. Chem. 86, 2229 (2014)

${ }^{30}$ S. Santesson, M. Andersson, E. Degerman, T. Johansson, J. Nilsson, and S. Nilsson, Anal. Chem. 72, 3412 (2000).

${ }^{31}$ H. Tanaka, Y. Wada, Y. Mizuno, and K. Nakamura, Jpn. J. Appl. Phys. 55, 067302 (2016).

${ }^{32}$ A. Marzo, T. Corkett, and B. W. Drinkwater, IEEE Trans. Ultrason. Ferroelectrics Freq. Control 65, 102 (2018).

${ }^{33}$ R. Barnkob, P. Augustsson, T. Laurell, and H. Bruus, Phys. Rev. E 86, 056307 (2012).

${ }^{34}$ D. M. Harris, T. Liu, and J. W. M. Bush, Exp. Fluids 56, 83 (2015). 
${ }^{35}$ M. A. B. Andrade, N. Perez, F. Buiochi, and J. C. Adamowski, IEEE Trans. Ultrason. Ferroelectrics Freq. Control 58, 1674 (2011).

${ }^{36}$ A. Stindt, M. A. B. Andrade, M. Albrecht, J. C. Adamowski, U. Panne, and J. Riedel, Rev. Sci. Instrum. 85, 015110 (2014).

${ }^{37}$ L. P. Gor'kov, Solviet Phys.-Dokl. 6, 773 (1962).

${ }^{38}$ L. E. Kinsler, A. R. Frey, A. B. Coppens, and J. V Sanders, Fundamentals of Acoustics (John Wiley \& Sons, New York, 1999).
${ }^{39}$ G. T. Silva and H. Bruus, Phys. Rev. E 90, 063007 (2014).

${ }^{40}$ M. A. B. Andrade, N. Pérez, and J. C. Adamowski, J. Acoust. Soc. Am. 136, 1518 (2014).

${ }^{41}$ N. Perez, M. A. B. Andrade, R. Canetti, and J. C. Adamowski, J. Appl. Phys. 116, 184903 (2014).

${ }^{42}$ T. Fushimi, T. L. Hill, A. Marzo, and B. W. Drinkwater, Appl. Phys. Lett. 113, 034102 (2018) 